\title{
Direct evidence of polar ferroelastic domain boundaries in semiconductor $\mathrm{BiVO}_{4}$
}

\author{
H. Yokota, ${ }^{1,2, a)}$ N. Hasegawa, ${ }^{1}$ M. Glazer,,${ }^{3,4}$ E. K. H. Salje, ${ }^{5}$ and Y. Uesu ${ }^{6}$ \\ ${ }^{1}$ Department of Physics, Chiba University, Chiba, 263-8522, Japan \\ 2 JST, PRESTO, Tokyo, 102-0076, Japan \\ ${ }^{3}$ Physics Department, University of Oxford, Oxford, OX1 3PU, U.K. \\ ${ }^{4}$ Department of Physics, University of Warwick, Coventry, CV4 7AL, U.K. \\ ${ }^{5}$ Department of Earth Sciences, Cambridge University, Cambridge, CB2 3EQ, U.K. \\ ${ }^{6}$ Department of Physics, Waseda University, Tokyo, 169-8555, Japan
}

Ferroelastic domain boundaries in semiconductor bismuth vanadate, $\mathrm{BiVO}_{4}$, are examined using second harmonic generation (SHG) microscopy. Although the bulk is centrosymmetric, domain boundaries produce homogeneous SH signals. The polarization dependences of SH intensities exhibite strong anisotropy compatible with the polar symmetry $m$. The present results are compared with the experimental results of other ferroelastics we have observed so far. Unlike other ferroelastic materials, the directions of the SH maxima are in the same direction for all domain boundaries.

Domain boundaries in ferroics are known to exhibit physical properties that do not exist in the bulk. Typical examples are domain boundaries with much higher conductivity than the bulk $^{1-6}$, even being superconducting ${ }^{7}$, charged domain boundaries ${ }^{8-15}$, and polar domain boundaries ${ }^{16-22}$. These phenomena have been directly measured by state-of-the-art techniques, such as aberration-corrected transmission microscopy, electron holography, atomic force microscopy etc. The fundamental significance of these phenomena lies in the possible development of new technologies where the domain boundary is the main element of the device. Racetrack memories ${ }^{23}$ and logics for electronic circuits ${ }^{24}$ are prominent examples of applications, and some of them have been already been achieved by using magnetic domain boundaries. Ferroelastic domain boundaries have several advantages for such future devices ${ }^{25}$. Since the width of a ferroelastic domain boundary is much thinner than any magnetic boundaries, a high-density memory device can be realized. In particular, the existence of polar properties at a ferroelastic domain boundary is one of the most promising candidates, because the localized polarization

${ }^{\text {a }}$ Contact email: hiroko.9bq@chiba-u.jp 
could be switchable without interfering with the depolarization field in the bulk. The polarity of domain boundaries has been reported in various oxides ${ }^{16-22}$ with different crystal structures, all of which are insulators in the bulk. To confirm the universality of the polar nature at a ferroelastic domain boundary, experiments using non-insulator materials are crucial. Since a semiconductor has a narrower bandgap than an insulator, it is possible to generate semiconductor devices based on the domain boundaries, independent of the properties of the bulk. A typical example is the role played by ferroelastic domain boundaries on the electronic properties of the high current density layer in $\mathrm{SrTiO}_{3} / \mathrm{LaAlO}_{3}$ sandwiches ${ }^{26}$. Thus, we have chosen bismuth vanadate $\left(\mathrm{BiVO}_{4}\right)$ because it is found to be a direct bandgap semiconductor by first-principle calculations and density functional theory (DFT) studies ${ }^{27}$.

$\mathrm{BiVO}_{4}$ has recently been the subject of considerable attention in the area of photocatalysis because of its high-performance for visible light-driven water splitting ${ }^{28}$. However, fundamental studies of domain boundaries in this material are lacking. The crystal structure of $\mathrm{BiVO}_{4}$ has three main crystal forms. Since the tetragonal zircon structure is only obtained by a low-temperature synthesis and the transformation process is irreversible, we only discuss here the scheelite structure. In this structure $\mathrm{BiVO}_{4}$ consists of isolated $\mathrm{VO}_{4}$ tetrahedra that are slightly elongated along the -4 axis. BiVO undergoes a ferroelastic phase transition at around $530 \mathrm{~K}$ from tetragonal $I 4_{1} / a$ to monoclinic $I 2 / a^{29-31}$. The $\mathrm{V}$ atom is fairly rigidly held within the oxygen tetrahedron. The $\mathrm{Bi}$ atoms are situated midway between the tetrahedra and are coordinated by eight $\mathrm{O}$ atoms. Below $530 \mathrm{~K}$ the -4 and $4_{1}$ symmetry elements of the paraelastic phase are lost, and the crystal symmetry changes from tetragonal to monoclinic. Apart from the symmetry change at the high-temperature phase transition, which is consistent with ferroelasticity, ferroelastic stress-strain hysteresis loops were actually observed by Lim and Jeong ${ }^{32}$. Second-harmonic generation (SHG) experiments have been performed using a powder sample and a single crystal below and above the ferroelastic phase transition temperature, but no SHG signal has been reported so far. Here, we present a study of domain boundaries in semiconductor $\mathrm{BiVO}_{4}$ using $\mathrm{SHG}$ microscopy. We show that the ferroelastic domain boundary in $\mathrm{BiVO}_{4}$ does exhibit polar properties.

The $\mathrm{BiVO}_{4}$ single crystal was grown by the Czochralski method ${ }^{30}$. The dimension of the specimen was $10 \times 12 \mathrm{~mm}^{2}$ and thickness $180 \mu \mathrm{m}$. It has a (010) cleavage plane with a two-fold axis perpendicular to the surface. Both surfaces were optically polished for SHG measurements because surface roughness has a negative influence on the experimental results. For the SHG measurements, an $\mathrm{Nd}: \mathrm{YVO}_{4}$ laser with a wavelength of $1064 \mathrm{~nm}$, repetition frequency of $40 \mathrm{kHz}$, pulse width of $10 \mathrm{~ns}$, laser energy of $3.125 \mu \mathrm{J}$ was used for the fundamental wave. An attenuator composed of a half-wave plate and a thin-film polarizer was used to precisely adjust the laser energy. The laser intensity was monitored by a photodetector. Since we adopt a scanning system with a piezo-actuators stage for lateral directions ( $X Y$ plane) and a stepping-motor for depth direction (Z-axis), it takes half an hour to obtain a two-dimensional (2D) image, depending on the step size. To reduce the effect of laser instability, the monitored laser intensity was used to normalize the SH intensity. The fundamental wave was focused on the sample by using an objective lens with a numerical aperture of 0.7 . The generated SH wave from the specimen was collected by an objective lens with a numerical aperture of 0.7 and detected by a photomultiplier tube. The SH signal obtained was synchronized with the fundamental wave through a lock-in amplifier. A polarizer was used to change the polarization direction of the fundamental wave and an analyzer for selecting a specific component of the $\mathrm{SH}$ wave.

Figure 1 shows the polarization microscope image under a crossed Nicol configuration. Only one type of 
ferroelastic domain boundary exists in this specimen. This domain boundary structure is quite similar to that in the previous report by David et $a l^{33}$. They observed the domain boundary formation as a function of temperature, and at room temperature only a few domain boundaries were observed. Our sample shows strong extinction, and its direction is parallel to the ferroelastic domain boundary. We selected a small area of $50 \times 5 \mu \mathrm{m}^{2}$ as in the enclosed white box in Fig. 1 and carried out the SHG microscope experiments. To avoid the surface effect producing relatively strong SH intensity even from centrosymmetric materials, we conducted a one-dimensional (1D) scan along the sample depth direction and chose the middle of the specimen as the focus position. Figure 2 (a) shows a 2D image of the SH wave distribution from a specimen. This image was taken with $0.2 \mu \mathrm{m}$ steps with the polarizer and analyzer directions parallel to each other. The bright colour represents the area with SH activity. SH-active regions appear as straight lines that are almost parallel to the $Y$-axis. This direction coincides well with the ferroelastic domain boundary. By comparing with a polarization microscope image, we can conclude that the SH active area corresponds to the ferroelastic domain boundary. The magnitude of the SH signal from $\mathrm{BiVO}_{4}$ is almost the same as with other ferroelastic materials ${ }^{19-22}$, and it is around $10^{-8}$ weaker than in normal ferroelectrics, which produce SH signals from the bulk. Except for ferroelastic domain boundaries, no SH signals are detected from the bulk region in $\mathrm{BiVO}_{4}$. This result suggests that $\mathrm{SH}$-activity appears only at the domain boundary while the bulk is centrosymmetric.

Several 2D scans were performed at different depths in order to construct a three-dimensional (3D) image of the SH wave distribution inside the sample ${ }^{34}$, and the result is shown in Fig. 2 (b). The magnitude of the SH intensity persists inside the specimen, and the domain boundaries are perpendicular to the cleavage plane. Since the $\mathrm{SH}$ intensity is homogeneous and also generated from the middle of the specimen, the possibilities of impurity or surface effects can be excluded as the origin of the SH-activity.

To clarify the polarity of the domain boundary, determination of the point group symmetry is essential because only 10 point groups belong to polar classes among the 20 point groups exhibiting SH-activity and piezo-activity. Our SHGM system allows us to determine the point group symmetry by measuring SH anisotropy. Because SHG is a second-order nonlinear optical phenomenon, the squared term in nonlinear polarization is responsible for the SHG process. This term is expressed as

$$
P_{i}=d_{i j k} E_{j} E_{k}
$$

Here $d_{i j k}$ is the SHG tensor component, and $E$ is the fundamental wave field. Since the tensor component is a representative of crystal symmetry, measuring SH anisotropy under different conditions gives us direct information about the point symmetry. We carried out SH anisotropy measurements under five different conditions; 

rotated every 6 degree,

(ii) A was rotated keeping P parallel to the domain boundary,

(iii) A was rotated keeping P perpendicular to the domain boundary,

(iv) P was rotated keeping A parallel to the domain boundary, and

(v) P was rotated keeping A perpendicular to the domain boundary.

We performed 2D scans under all configurations, and the polarization dependence of the SH intensity was plotted for each scanning point. Figure 3 shows a polar diagram mapped ${ }^{35}$ under condition (i). Apart from the domain boundary, the SH intensity is almost zero from the bulk under any polarization directions. Although the magnitudes of the SH intensity are different, the shapes of the $\mathrm{SH}$ anisotropies are similar for all domain boundaries. They exhibit SH maxima in the same direction. This tendency is different from other ferroelastic materials where polarity has been confirmed at a domain boundary. For example, in $\mathrm{CaTiO}_{3}$ and $\mathrm{Pb}_{3}\left(\mathrm{PO}_{4}\right)_{2}^{19,22}$, we observed that the orientations of $\mathrm{SH}$ maxima are slightly different for each domain boundary, especially when the density of domain boundaries is high. We believe that the static interaction between polar domain boundaries plays some contribution in this phenomena. It is also interesting to notice the difference between semiconducting $\mathrm{BiVO}_{4}$ like and pure insulators like $\mathrm{CaTiO}_{3}$ and $\mathrm{Pb}_{3}\left(\mathrm{PO}_{4}\right)_{2}$, where free carriers contribute differently to the formation of polarity in domain boundaries. Figure 4 summarizes the polarization dependence of the SH intensity under the five different configurations. These plots are representative of each SH anisotropy and were selected from the same domain boundary. Figure 4 (a) is the result of condition (i). The SH maximum was obtained at a direction $20^{\circ}$ away from the domain boundary orientation. It has an additional local maximum, and the angle between the maximum and local maximum is around $70^{\circ}$. Figure 4 (b) and (c) show polar diagrams for conditions (ii) and (iii), respectively. Both anisotropies exhibit the same profiles with different intensity. The result of condition (ii) is not as smooth as that of condition (iii) as a result of a weaker signal. The intensity ratio between (ii) and (iii) was $1: 7.5$. Both SH anisotropies exhibit SH maxima in the direction parallel to the domain boundary. Figure 4 (d) and (e) are the results of conditions (iv) and (v). In the case of (iv), it shows an SH maximum at $180^{\circ}$ which is almost perpendicular to the cases of (ii) and (iii). For (v), the shape of SH anisotropy is different from (iv), and it has a local maximum. The magnitude of SH intensity for (v) is weaker than that of (iv) with a $1: 7$ ratio.

Ferroelastic domain boundaries maintain strain compatibility between neighboring domains. Its orientation was theoretically determined based on the criterion proposed by Fousek and Janovec ${ }^{36}$ and depends on the crystal symmetries of prototypic and ferroelastic phases ${ }^{37}$. For $\mathrm{BiVO}_{4}$, the ferroelastic phase transition can be expressed as $4 / m F 2 / m$ using the 
notation of $\mathrm{Aizu}^{38}$. Two domain boundaries appear as a result of this structural phase transition, and the equations of these domain boundary planes are expressed as

$$
\begin{aligned}
& x=p y \\
& x=-y / p
\end{aligned}
$$

with $p=\frac{b+\sqrt{a^{2}+b^{2}}}{a}$. Here, $a$ and $b$ are the strain tensor components. These two domain boundaries belong to crystallographically non-prominent $W^{\prime}$-wall, and they are perpendicular to each other. Since the cleavage plane of $\mathrm{BiVO}_{4}$ is (010) the monoclinic plane contains a mirror plane, and the domain boundaries are perpendicular to the cleavage plane. This is consistent with our SHG 3D observations, because SH-active planes are perpendicular to the sample surface. To explain the SH-activity, we assume that the symmetry of the domain boundary is a subgroup of $4 / \mathrm{m}$ and attempt to fit the data using point group $m$. The SHG $d$ tensor component for point group $m$ can be described ${ }^{39}$ using Voigt notation as

$$
\left(\begin{array}{cccccc}
d_{11} & d_{12} & d_{13} & 0 & d_{15} & 0 \\
0 & 0 & 0 & d_{24} & 0 & d_{26} \\
d_{31} & d_{32} & d_{33} & 0 & d_{35} & 0
\end{array}\right) .
$$

Here, the monoclinic axis is parallel to the $x_{2}$ axis, which means the mirror plane is perpendicular to this axis. When we assume that the orientations of the principal axes for the domain boundary are restricted by those of the bulk and keep the same directions, only six SHG tensor components, which do not contain $x_{2}$, affect the $\mathrm{SH}$ anisotropy. The induced nonlinear polarization is then expressed by

$$
\begin{aligned}
& P_{1} \propto d_{11} E_{1}^{2}+d_{13} E_{3}^{2}+d_{15} E_{1} E_{3}, \\
& P_{3} \propto d_{31} E_{1}^{2}+d_{33} E_{3}^{2}+d_{35} E_{1} E_{3}, \\
& E_{1}=E_{0} \sin (\theta+\alpha) \\
& E_{3}=E_{0} \cos (\theta+\alpha),
\end{aligned}
$$

where $E_{0}$ is the electric field of the fundamental wave. In the above equations, $\theta$ is the rotation angle of the polarizer from the $X$-axis and $\alpha$ is the angle between the $X$-axis and the $x_{3}$ axis. The SH intensity after passing through the analyzer will be

$$
I \propto P^{2} \propto\left\{P_{1} \sin (\varphi+\alpha)+P_{3} \cos (\varphi+\alpha)\right\}^{2} .
$$

Here $\varphi$ is the rotation angle of the analyzer from the $X$-axis. The experimental results were fitted using eq. (8) and the results are summarized in Figs. 4. During the fitting process, parameters representing each independent component were restricted to the same values for all five configurations. The experimental results are well reproduced by point group $m$ except for 
condition (v). In this case, the fitted result is not as good as the other conditions because of its rather weak intensity. However, it represents some variations of the direction of the $\mathrm{SH}$ maximum and the existence of a local maximum. As a result of the fitting procedure, the relative magnitude of the $d$ tensor components are obtained as $d_{11}: d_{13}: d_{15}: d_{31}: d_{33}: d_{35}=1:-79:$ $-6.5: 2: 9.5: 10$. For point group $m$, the polarization is in the mirror plane, but there is no symmetry restriction within a mirror plane. While it is difficult to determine the exact orientation of the polarization, SH anisotropy gives us some idea about it. It is known that the magnitude of the SHG tensor component is empirically proportional to the spontaneous polarization. Therefore, in $\mathrm{BiVO}_{4}$ the polarization appearing at the domain boundary should be almost parallel to the domain boundary as was generally found in model simulations ${ }^{40,41}$. Further experiments using different experimental techniques are required to confirm this result.

Ferroelastic domain boundaries in semiconductor $\mathrm{BiVO}_{4}$ were examined by a $\mathrm{SHG}$ microscope. SH-activity was observed at the domain boundary, similar to that found in insulator ferroelastics. Compared with other ferroelastic materials, whose domain boundaries are already confirmed as polar, neighboring polarizations are less interacting and so they are aligned in the same direction for each domain boundary. The polarization dependence of the SH intensity shows strong anisotropy, and this is consistent with the symmetry of point group $m$, which belongs to a polar class. It is also estimated that the semiconductor nature of this crystal produces a reduction of domain boundary interactions, which could make easy the position control of domain boundaries.

H. Y. is grateful to the Ministry of Education, Culture, Sports, Science and Technology in Japan for financial support (Grant No. 17K05489) and JST PRESTO Grand number JPMJPR19LA, Japan and the Iketani Science and Technology Foundation (Grant No. 0311045-A)

DATA AVAILABLITY STATEMENTS

The data that support the findings of this study are available from the corresponding author upon reasonable request. 


\section{REFERENCES}

${ }^{1}$ J. Seidel, L.W. Martin, Q. He, Q. Zhan, Y.-H. Chu, A. Rother, M.E. Hawkridge, P. Maksymovych, P. Yu, M. Gajek, N. Balke, S. V. Kalinin, S. Gemming, F. Wang, G. Catalan, J.F. Scott, N.A. Spaldin, J. Orenstein, and R. Ramesh, Nat. Mater. 8, 229 (2009).

${ }^{2}$ T. Sluka, A.K. Tagantsev, P. Bednyakov, and N. Setter, Nat. Commun. 4, 1808 (2013).

${ }^{3}$ J. Seidel, P. Maksymovych, Y. Batra, A. Katan, S.-Y. Yang, Q. He, A.P. Baddorf, S. V. Kalinin, C.-H. Yang, J.-C. Yang, Y.-H. Chu, E.K.H. Salje, H. Wormeester, M. Salmeron, and R. Ramesh, Phys. Rev. Lett. 105, 197603 (2010).

${ }^{4}$ Y. Kim, M. Alexe, and E.K.H. Salje, Appl. Phys. Lett. 96, 032904 (2010).

${ }^{5}$ P. Maksymovych, J. Seidel, Y.H. Chu, P. Wu, A.P. Baddorf, L.-Q. Chen, S. V. Kalinin, and R. Ramesh, Nano Lett. 11, 1906 (2011).

${ }^{6}$ S. Farokhipoor and B. Noheda, Phys. Rev. Lett. 107, 127601 (2011).

${ }^{7}$ A. Aird and E.K.H. Salje, J. Phys. Condens. Matter 10, L377 (1998).

${ }^{8}$ B.M. Vul, G.M. Guro, and I.I. Ivanchik, Ferroelectrics 6, 29 (1973).

${ }^{9}$ C.-L. Jia, S.-B. Mi, K. Urban, I. Vrejoiu, M. Alexe, and D. Hesse, Nat. Mater. 7, 57 (2008).

${ }^{10}$ N. Balke, M. Gajek, A.K. Tagantsev, L.W. Martin, Y.-H. Chu, R. Ramesh, and S. V. Kalinin, Adv. Funct. Mater. 20, 3466 (2010).

${ }^{11}$ Y. Du, X.L. Wang, D.P. Chen, S.X. Dou, Z.X. Cheng, M. Higgins, G. Wallace, and J.Y. Wang, Appl. Phys. Lett. 99, 252107 (2011).

${ }^{12}$ P. Zubko, S. Gariglio, M. Gabay, P. Ghosez, and J.-M. Triscone, Annu. Rev. Condens. Matter Phys. 2, 141 (2011).

${ }^{13}$ Y. Qi, Z. Chen, C. Huang, L. Wang, X. Han, J. Wang, P. Yang, T. Sritharan, and L. Chen, J. Appl. Phys. 111, 104117 (2012)

${ }^{14}$ W. Wu, Y. Horibe, N. Lee, S.-W. Cheong, and J.R. Guest, Phys. Rev. Lett. 108, 077203 (2012).

${ }^{15}$ L. Li, P. Gao, C. Nelson, Y. Zhang, S.-J. Kim, A. Melville, C. Adamo, D. Schlom, and X. Pan, Microsc. Microanal. 19, 1654 (2013)

${ }^{16}$ J.F. Scott, E.K.H. Salje, and M.A. Carpenter, Phys. Rev. Lett. 109, 187601 (2012).

${ }^{17}$ S. Van Aert, S. Turner, R. Delville, D. Schryvers, G. Van Tendeloo, and E.K.H. Salje, Adv. Mater. 24, 523 (2012). 
${ }^{18}$ E.K.H. Salje, O. Aktas, M.A. Carpenter, V. V. Laguta, and J.F. Scott, Phys. Rev. Lett. 111, 247603 (2013).

${ }^{19}$ H. Yokota, H. Usami, R. Haumont, P. Hicher, J. Kaneshiro, E.K.H. Salje, and Y. Uesu, Phys. Rev. B - Condens. Matter Mater. Phys. 89, 144109 (2014)

${ }^{20}$ H. Yokota, S. Niki, R. Haumont, P. Hicher, and Y. Uesu, AIP Adv. 7, 085315 (2017).

${ }^{21}$ H. Yokota, S. Matsumoto, E.K.H. Salje, and Y. Uesu, Phys. Rev. B 98, 104105 (2018).

${ }^{22}$ H. Yokota, S. Matsumoto, E.K.H. Salje, and Y. Uesu, Phys. Rev. B 100, 024101 (2019).

${ }^{23}$ S.S.P. Parkin, M. Hayashi, and L. Thomas, Science (80-. ). 320, 190 (2008).

${ }^{24}$ D.A. Allwood, Science (80-. ). 309, 1688 (2005).

${ }^{25}$ E.K.H. Salje, ChemPhysChem 11, 940 (2010).

${ }^{26}$ Y. Frenkel, N. Haham, Y. Shperber, C. Bell, Y. Xie, Z. Chen, Y. Hikita, H.Y. Hwang, E.K.H. Salje, and B. Kalisky, Nat. Mater. 16, 1203 (2017).

${ }^{27}$ A. Walsh, Y. Yan, M.N. Huda, M.M. Al-Jassim, and S.H. Wei, Chem. Mater. 21, 547 (2009).

${ }^{28}$ A. Kudo, K. Ueda, H. Kato, and I. Mikami, Catal. Letters 53, 229 (1998).

${ }^{29}$ W.I.F. David, A.M. Glazer, and A.W. Hewat, Phase Transitions 1, 155 (1979).

${ }^{30}$ A.W. Sleight, H. -y. Chen, A. Ferretti, and D.E. Cox, Mater. Res. Bull. 14, 1571 (1979).

${ }^{31}$ J.D. Bierlein and A.W. Sleight, Solid State Commun. 16, 69 (1975).

${ }^{32}$ Ae Ran Lim and S.-Y. Jeong, J. Intell. Mater. Syst. Struct. 21, 915 (2010).

${ }^{33}$ W.I.F. David and I.G. Wood, J. Phys. C Solid State Phys. 16, 5149 (1983).

${ }^{34}$ J. Kaneshiro, S. Kawado, H. Yokota, Y. Uesu, and T. Fukui, J. Appl. Phys. 104, 054112 (2008).

${ }^{35}$ J. Kaneshiro and Y. Uesu, Phys. Rev. B 82, 184116 (2010).

${ }^{36}$ J. Fousek and V. Janovec, J. Appl. Phys. 40, 135 (1969).

${ }^{37}$ J. Sapriel, Phys. Rev. B 12, 5128 (1975).

${ }^{38}$ K. Aizu, J. Phys. Soc. Japan 28, 706 (1970).

${ }^{39}$ J.F. Nye, Physical Properties of Crystals : Their Representation by Tensors and Matrices (Clarendon Press, Oxford, 1985). 
${ }^{40}$ E.K.H. Salje, S. Li, M. Stengel, P. Gumbsch, and X. Ding, Phys. Rev. B 94, 024114 (2016).

${ }^{41}$ L. Goncalves-Ferreira, S.A.T. Redfern, E. Artacho, and E.K.H. Salje, Phys. Rev. Lett. 101, 097602 (2008).

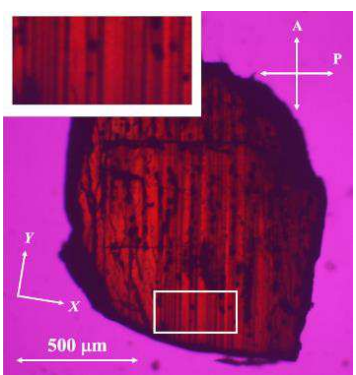

FIG. 1 (Colour online) A polarization microscope image under a crossed Nicol configuration. White arrows indicate the directions of the polarizer and analyzer. The axes of the coordinate system is shown marked as $X$ and $Y$ directions. An expanded image is shown as an inset. Only one type of ferroelastic domain boundary parallel to the analyzer direction is observed. Irregular dark lines are scratches or irregularities on the surface. These do not affect the SHG observations.

(a)
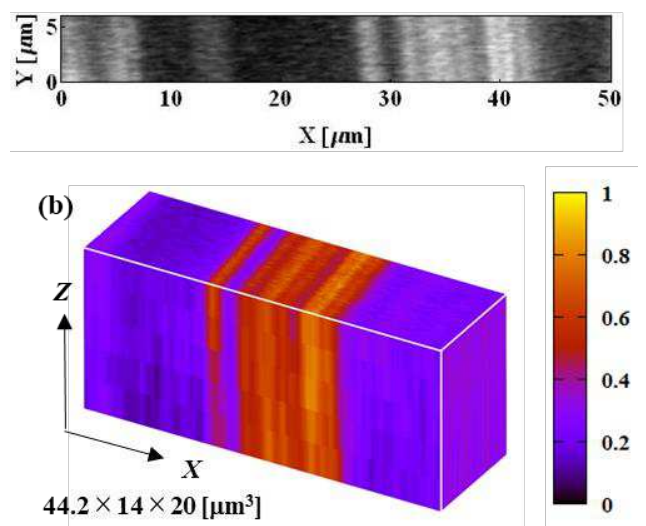

FIG. 2 (Colour online) SHG images of $\mathrm{BiVO}_{4}$. (a) shows a 2D image with $0.2 \mu \mathrm{m}$ scanning steps. Stronger SH intensity is detected from the area with brighter colour. These SH-active areas appear as straight lines that correspond to the direction of the ferroelastic domain boundary. (b) shows the constructed 3D image of SH wave distributions. SH activity remains inside the sample. The colour bar corresponds to the magnitude of SH intensity. 


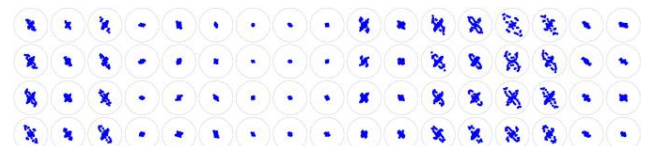

FIG. 3 (Colour online) Polar diagram of $\mathrm{BiVO}_{4}$ under the polarization directions of polarizer and analyzer being parallel to each other. No SH signal is detected from the bulk region, and only the ferroelastic domain boundary exhibits SH-activity. The directions of SH maxima are the same for all domain boundaries that are parallel to the domain boundary.

(a)

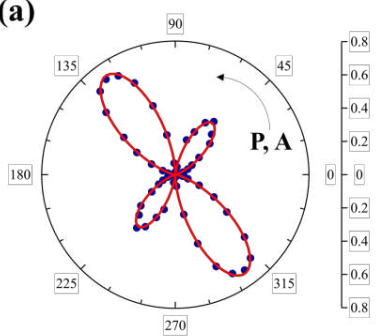

(b)

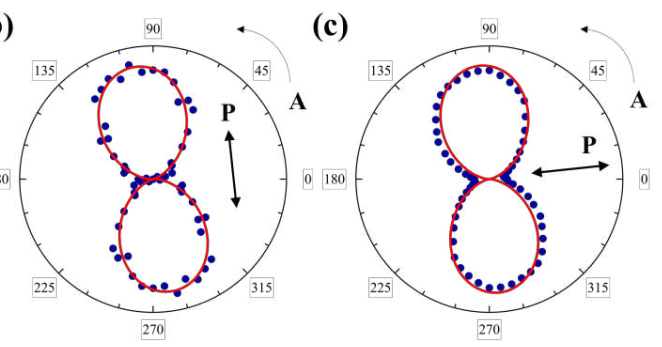

(d)

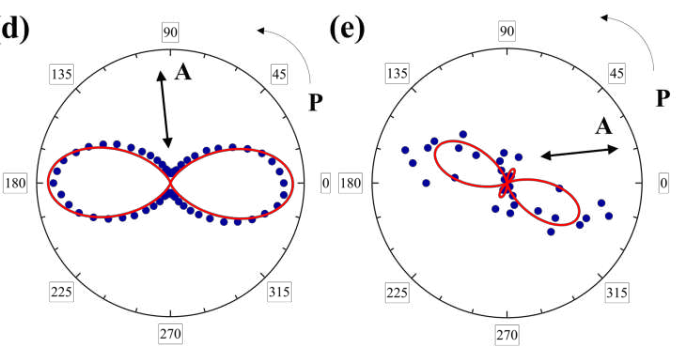

FIG. 4 (Colour online) Polarization dependence of SH intensity under different configurations. (a) the polarization directions of polarizer and analyzer being parallel to each other, (b) the polarization direction of the polarizer being parallel to the domain boundary, (c) the polarization direction of the polarizer being perpendicular to the domain boundary, (d) the 
polarization direction of the analyzer being parallel to the domain boundary and (e) the polarization direction of the analyzer being perpendicular to the domain boundary. The arrows indicate the directions of fixed polarizer (P) or analyzer (A) . The circle indicates the rotation of $\mathrm{P}$ or $\mathrm{A}$. 


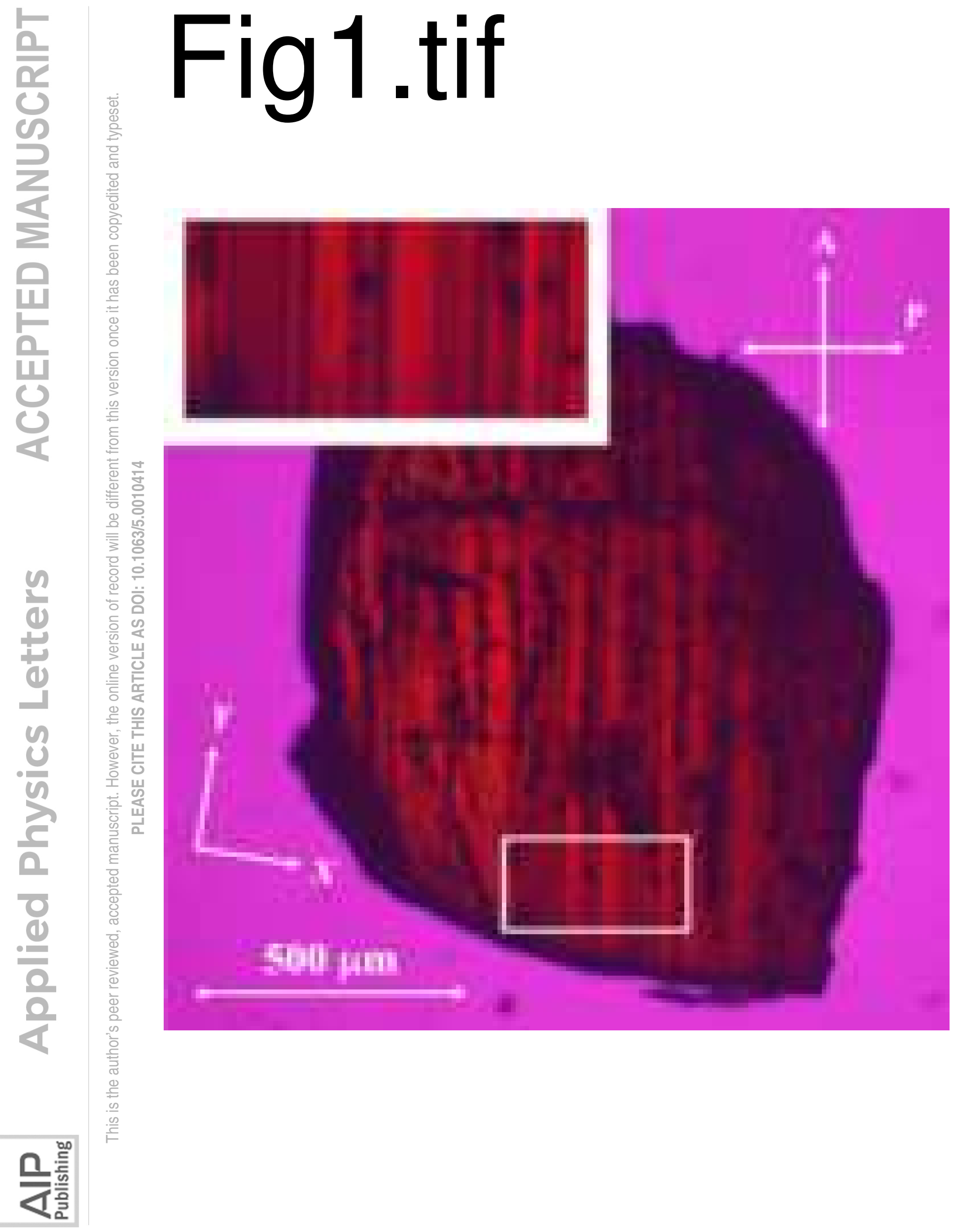




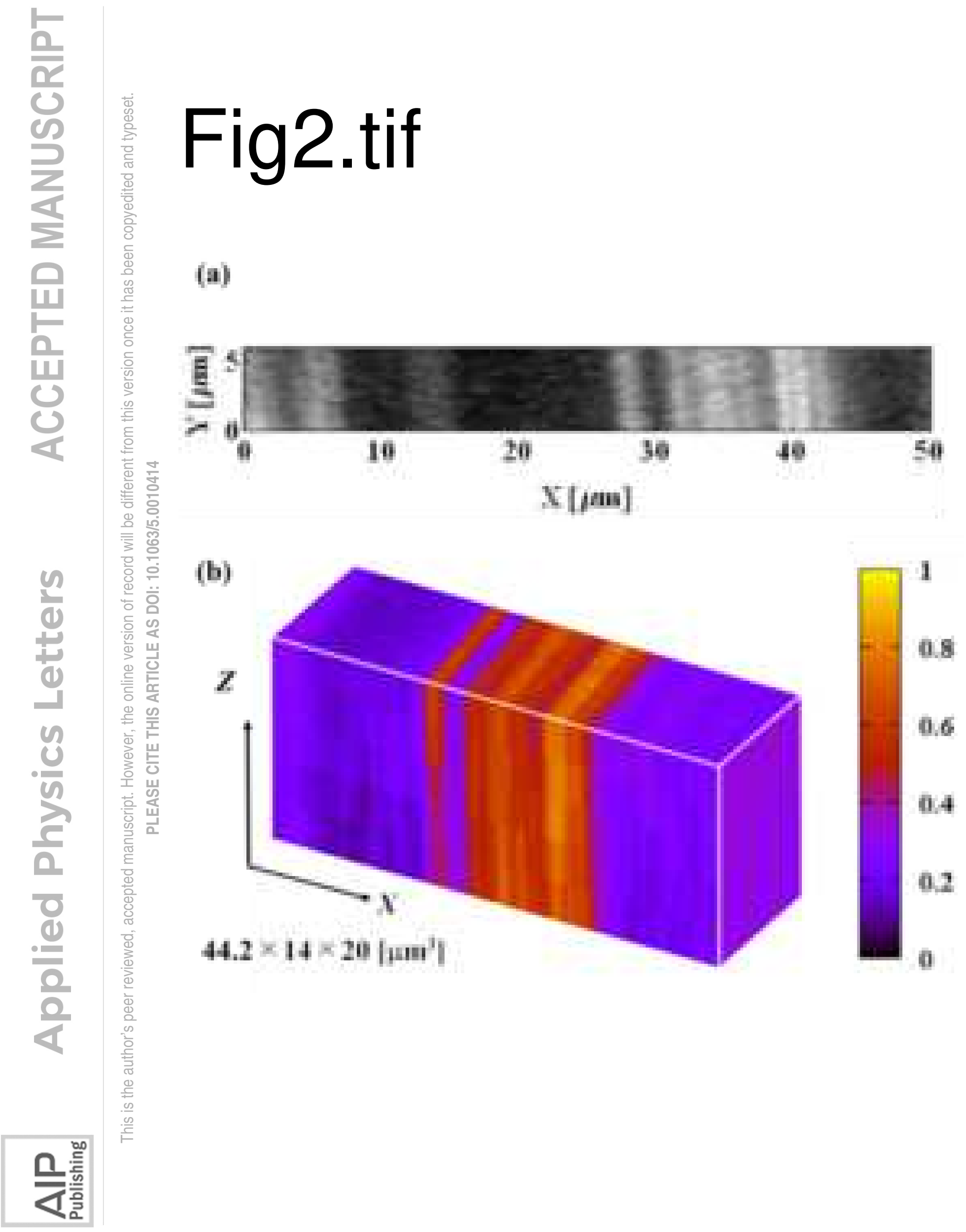




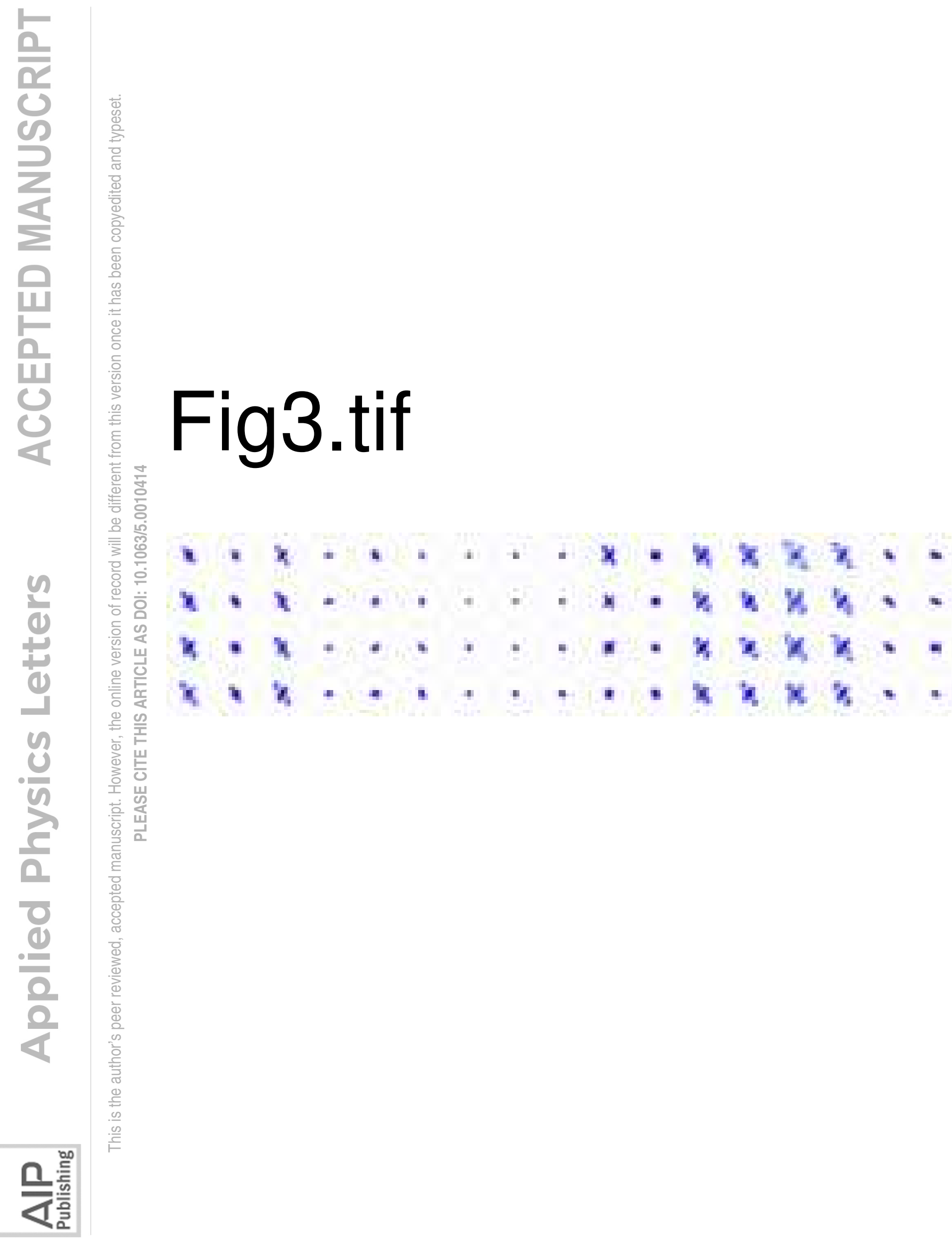




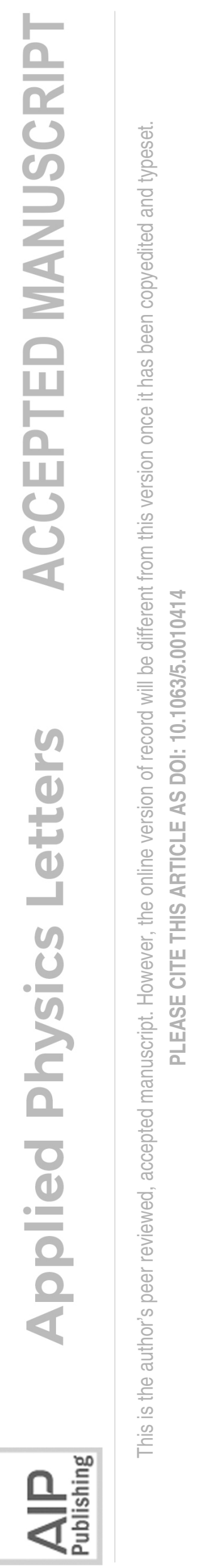

\section{Fig4_2.tif}

(a)
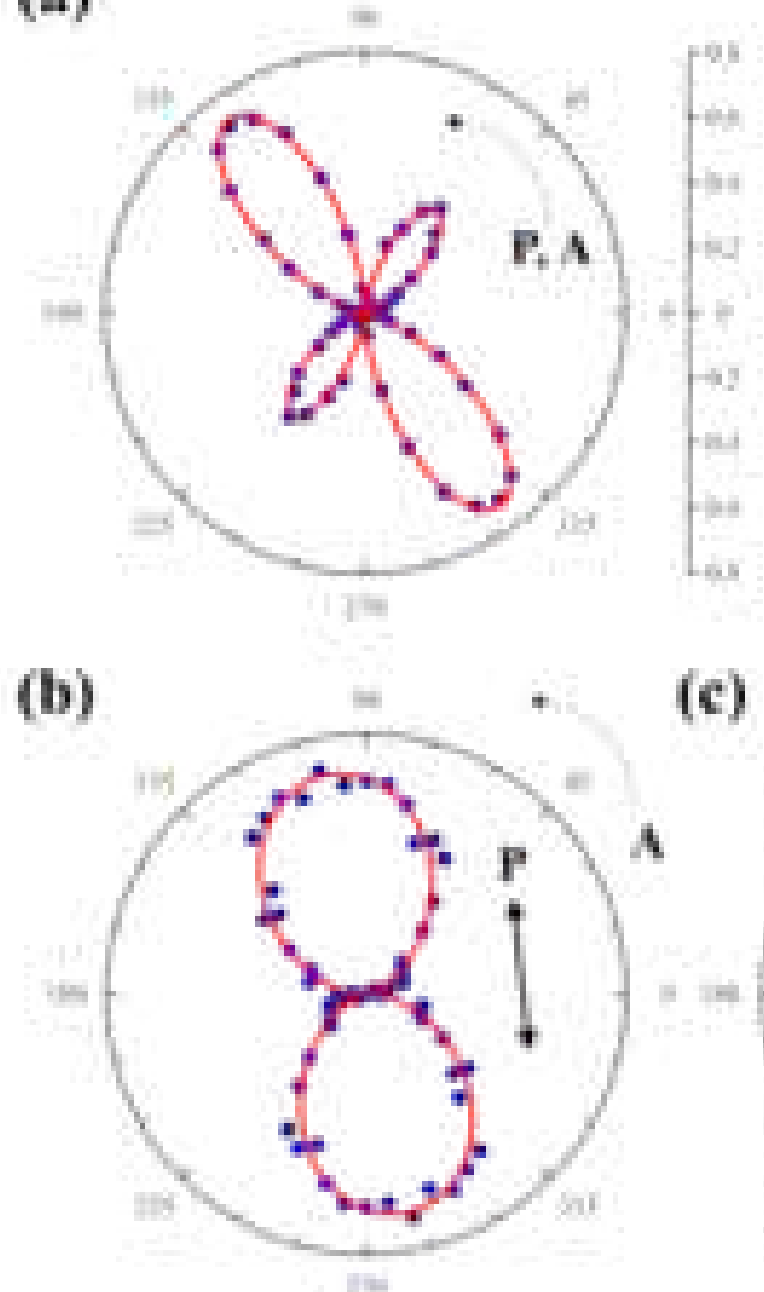

(c)

(d)

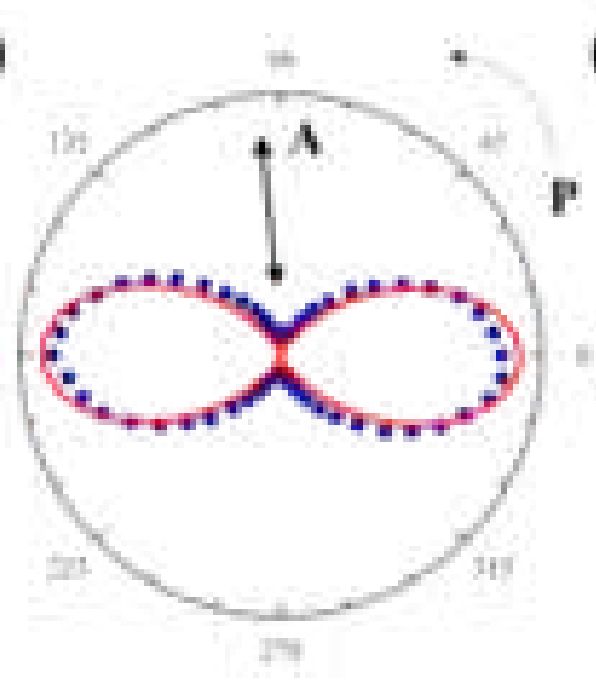

(e)

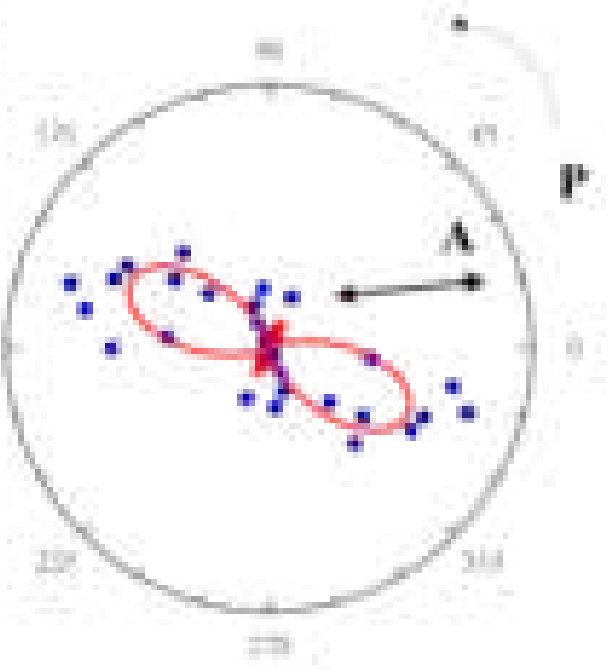

